\title{
Potencial antileishmania, antimalárico e antitrypanossoma de espécies de Casearia: Uma revisão integrativa
}

\author{
Antileishmania, antimalarial and antitrypanosome potential of Casearia species: An integrative \\ review
}

Antileishmania, potencial antipalúdico y antitrypanosoma de las especies de Casearia: Una revisión integradora

\section{Resumo}

O tratamento de doenças parasitárias tem como um de seus maiores desafios a resistência desenvolvida pelos parasitos contra os medicamentos disponíveis. Portanto, a busca por alternativas terapêuticas é urgente. Este estudo realizou uma revisão integrativa da literatura que avaliou o potencial antileishmania, antimalárico e antitrypanossoma de plantas do gênero Casearia. A busca dos artigos científicos foi realizada no Portal de Periódicos da CAPES (PPC), Biblioteca Virtual em Saúde (BVS), PUBMED e SCIELO, utilizando-se os seguintes descritores: Casearia e antileishmania; Casearia e antimalárico; e Casearia e antitrypanossoma. Um total de 122 publicações foram coletadas para triagem, destes, 11 foram incluídos na revisão integrativa. Na atividade antileishmania, a Casearia sylvestris se destacou, com extrato hexano da madeira e casca do caule e o extrato etanólico da casca da raiz apresentando atividade contra diferentes espécies de Leishmania $\left(\mathrm{CI}_{50}<100 \mu \mathrm{g} / \mathrm{mL}\right)$, porém com elevada toxicidade $\left(\mathrm{CC}_{50}<100\right.$ $\mu \mathrm{g} / \mathrm{mL}$ ). Dentre as substâncias isoladas, o flavonoide Tricina se mostrou ativo contra Leishmania e não citotóxico. Quanto a atividade antimalárica, 11 extratos de 3 espécies, C. elliptica, C. coriaceae e C. sylvestris mostraram-se ativos $\left(\mathrm{CI}_{50}<100 \mu \mathrm{g} / \mathrm{mL}\right)$, no entanto, apresentaram toxicidade. $\mathrm{Na}$ atividade antitrypanossoma, as casearinas isoladas das folhas de $C$. sylvestris, apresentaram grande potencial contra a forma tripomastigota de $T$. cruzi $\left(\mathrm{CI}_{50}<3,0 \mu \mathrm{g} / \mathrm{mL}\right)$, porém, apresentaram elevada toxicidade. As espécies de Casearia são, portanto, promissoras quanto à sua atividade antiparasitária, mas sua toxicidade é um problema. No entanto, os flavonoides da espécie apresentam bom índice de seletividade e, alterações moleculares e/ou formulações adequadas podem reduzir a toxicidade dos outros componentes.

Palavras-chave: Casearia; Leishmania; Malária; Doença de Chagas.

\begin{abstract}
One of the greatest challenges in the treatment of parasitic diseases is the resistance developed by the parasites against the available drugs. Therefore, the search for therapeutic alternatives is urgent. This study carried out an integrative review of the literature that evaluated the antileishmanial, antimalarial and antitrypanosome potential of plants of the genus Casearia. The search for scientific articles was carried out on the Portal of Journals from CAPES, Biblioteca Virtual em Saúde (BVS), PUBMED and SCIELO, using the following descriptors: Casearia and antileishmania; Casearia and antimalárico; and Casearia and antitrypanosoma. A total of 122 publications were collected for
\end{abstract}


screening, of which 11 were included in the integrative review. In the antileishmania activity, Casearia sylvestris stood out, with the hexane extract of the wood and stem bark and the ethanolic extract of the root bark showing activity against different species of Leishmania $\left(\mathrm{IC}_{50}<100 \mu \mathrm{g} / \mathrm{mL}\right)$, but with high toxicity $\left(\mathrm{CC}_{50}<100 \mu \mathrm{g} / \mathrm{mL}\right)$. Among the isolated substances, the flavonoid Tricina was shown to be active against Leishmania and non-cytotoxic. Regarding antimalarial activity, 11 extracts from 3 species, C. elliptica, C. coriaceae and $C$. sylvestris were shown to be active $\left(\mathrm{IC}_{50}<100 \mu \mathrm{g} / \mathrm{mL}\right)$, however, they showed high toxicity. In the antitrypanosome activity, casearins isolated from the leaves of C. sylvestris, showed great potential against the trypomastigote form of $T$. cruzi $\left(\mathrm{IC}_{50}<3.0 \mu \mathrm{g} / \mathrm{mL}\right)$, however, they showed high toxicity. Casearia species are therefore promising in terms of their antiparasitic activity, but their toxicity is a problem. However, the flavonoids of the species have a good selectivity index and, molecular alterations and/or adequate formulations can reduce the toxicity of the other components.

Keywords: Casearia; Leishmania; Malaria; Chagas Disease.

\section{Resumen}

Uno de los mayores desafíos en el tratamiento de las enfermedades parasitarias es la resistencia que desarrollan los parásitos frente a los fármacos disponibles. Por tanto, la búsqueda de alternativas terapéuticas es urgente. Este estudio realizó una revisión integradora de la literatura que evaluó el potencial antileishmanial, antipalúdico y antitrypanosoma de plantas del género Casearia. La búsqueda de artículos científicos se realizó en el Portal de Revistas de CAPES, Biblioteca Virtual em Saúde (BVS), PUBMED y SCIELO, utilizando los siguientes descriptores: Casearia y antileishmania; Casearia y antimalárico; e Casearia y antitrypanossoma. Se recopilaron 122 publicaciones para su cribado, de las cuales 11 se incluyeron en la revisión integradora. En la actividad antileishmania se destacó Casearia sylvestris, con el extracto de hexano de la madera y corteza del tallo y el extracto etanólico de la corteza de la raíz mostrando actividad frente a diferentes especies de Leishmania $\left(\mathrm{CI}_{50}<100 \mu \mathrm{g} / \mathrm{mL}\right)$, pero con alta toxicidad $\left(\mathrm{CC}_{50}<100 \mu \mathrm{g} / \mathrm{mL}\right)$. Entre las sustancias aisladas, se demostró que el flavonoide Tricina es activo contra Leishmania y no citotóxico. En cuanto a la actividad antipalúdica, 11 extractos de 3 especies, C. elliptica, $C$. coriaceae y $C$. sylvestris se mostraron activos $\left(\mathrm{CI}_{50}<100 \mu \mathrm{g} / \mathrm{mL}\right)$, sin embargo, mostraron alta toxicidad. En la actividad antitrypanosómica, las casearinas aisladas de las hojas de C. sylvestris, mostraron gran potencial contra la forma tripomastigota de $T$. cruzi $\left(\mathrm{CI}_{50}<3.0 \mu \mathrm{g} / \mathrm{mL}\right)$, sin embargo, mostraron alta toxicidad. Por lo tanto, las especies de Casearia son prometedoras en términos de su actividad antiparasitaria, pero su toxicidad es un problema. Sin embargo, los flavonoides de la especie tienen un buen índice de selectividad y las alteraciones moleculares y/o formulaciones adecuadas pueden reducir la toxicidad de los demás componentes.

Palabras clave: Casearia; Leishmania; Paludismo; Enfermedad de Chagas.

\section{Introdução}

As doenças infecciosas parasitárias representam um importante problema de saúde pública sendo uma das principais causas de morte no Brasil e no mundo, com cerca de 500 mil mortes anuais (Waldman; Sato, 2016; WHO, 2021). Embora essas doenças representem um problema de saúde pública global, atingindo principalmente países onde parte significativa da população vive em condições vulneráveis, a indústria farmacêutica demonstra desinteresse em desenvolver novos fármacos, tendo em vista que, a população atingida é de baixa renda e o retorno lucrativo seria insignificante (Brasil, 2010). Neste cenário, algumas infecções parasitárias como malária, leishmaniose e doença de chagas se destacam, prejudicando de forma permanente a saúde da população (Waldman; Sato, 2016).

As leishmanioses são um grupo de doenças infecciosas transmitidas através de vetores causadas por parasitas obrigatórios do gênero Leishmania, afetam pele e membranas mucosas, e são transmitidas para humanos pela picada de flebotomíneo (Alvar et al., 2012). Estima-se que ocorra de 700.000 a 1.000 .000 de novos casos por ano e que 20.000 a 30.000 casos evoluem a óbito anualmente (WHO, 2019). A malária também é uma doença parasitária protozoária, porém, causada por parasitas do gênero Plasmodium, transmitida pela fêmea do mosquito Anopheles (WHO, 2017). Sintomas associados são inespecíficos e no geral incluem calafrios, dor de cabeça, náuseas e dores musculares (Brasil, 2007; França et al., 2008). Mesmo que existam alternativas para evitar e tratar a malária, no ano de 2018, houveram em torno de 229 milhões de casos da doença espalhados no mundo (WHO, 2019).

Quanto a doença de Chagas, esta também é uma doença infecciosa, causada pelo protozoário Trypanosoma cruzi, transmitida através de vetores que são os triatomíneos hematófagos (BRASIL, 2017). Em quadros graves da doença ocorre comprometimento do sistema cardíaco e digestivo (Rassi et al., 2010). Estima-se um alcance de 6 a 7 milhões de pessoas 
infectadas a nível mundial e que 80 milhões de pessoas possuem o risco de contrair a doença, com número de mortes anuais estimado em 7 mil. No Brasil, estima-se que existam mais de 1 milhão de casos de pessoas infectadas pela doença (WHO, 2016).

Os medicamentos disponíveis para o tratamento destas doenças apresentam sérios problemas em termos de segurança, resistência, estabilidade e custos (DNDI, 2018). O alto custo agregado ao processo de pesquisa e desenvolvimento de novos fármacos, aliado ao baixo retorno financeiro esperado nos casos destas doenças, leva à quase ausência de novos medicamentos. Entre os anos de 1975 e 1999, somente 1\% dos 1.393 fármacos registrados foram destinados a doenças parasitárias (Sobrinho et al., 2007). Os fármacos existentes podem causar diversos efeitos colaterais graves como: sintomas de hipersensibilidade, depressão da medula óssea e polineuropatia periférica (Dias, Coura, 1997). Certo grau de insuficiência renal e ototoxicidade, além da cardiotoxicidade grave, levando à morte de indivíduos (DNDI, 2018).

Além dos problemas relacionados à segurança dos medicamentos atualmente disponíveis, os casos de resistência dos parasitos têm aumentado (Soeira; Castro, 2009), o que representa uma ameaça ao controle destas doenças. Levando-se em consideração que estas doenças assolam diversos países no mundo, é notável a necessidade urgente de novas alternativas terapêuticas.

Dentre as possíveis fontes de novos ativos para medicamentos, as plantas medicinais estão entre as mais importantes. Nesse contexto, o Brasil é privilegiado por possuir a maior biodiversidade do mundo, com mais de 56 mil espécies de plantas (Brasil, 1998), dentre elas, uma família tem se destacado devido ao amplo uso medicinal, a Salicaceae, que engloba espécies com atividades biológicas de grande importância, como: antitérmica, anestésica, antimicrobiana, antiprotozoária, antidiarreica, antiofídica, antirreumática, antisséptica, cicatrizante, anti-inflamatória (Junges et al., 1985; Silva et al., 2008) antioxidante, atividades citotóxicas, entre outras (Mosaddik et al., 2004).

O gênero Casearia, pertencente à família Salicaceae, está representado por mais de 180 espécies descritas em literatura, das quais 70 encontram-se distribuídas no continente americano e 37 estão presentes no Brasil (Marquete, 2007). Cerca de 287 compostos já foram identificados neste gênero, e pode-se afirmar que os terpenóides são a classe de metabólitos prevalente, em especial diterpenos clerodânicos. Também há relatos de fenilpropanoides, esteroides, flavonoides e outros fenólicos (Xia et al., 2014).

\section{Métodos}

Para realização desta revisão integrativa, foi desenvolvida uma pesquisa de seleção de artigos científicos disponíveis nas seguintes plataformas de busca: Portal de Periódicos CAPES (PPC), Biblioteca Virtual em Saúde (BVS), Biblioteca Nacional de Medicina (PUBMED) e Biblioteca Eletrônica Cientifica Online (SCIELO), não sendo delimitado o ano de publicação dos artigos. O período de busca foi entre janeiro à março de 2021, sendo adotados como critérios de inclusão apenas artigos em português, inglês e espanhol, bem como adequação do título ao tema analisado, e resumo compatível. Em relação aos critérios de exclusão, foram adotados os seguintes: artigos que não abordassem o tema de estudo, artigos de outros idiomas, artigos que testaram extratos e frações obtidas de outras espécies de plantas, artigos não disponíveis na íntegra, duplicados, e artigos que não foram analisados por especialistas.

Os descritores representativos para a pesquisa dos artigos nas plataformas de busca foram: Casearia e antileishmania, Casearia e antimalárico e Casearia e antitrypanossoma. Inicialmente, foram coletados 122 para triagem (PPC = 99, BVS=11, PUBMED = 9 e SCIELO = 3), entretanto, deste total, após análise do título e resumo, foram excluídos 106 artigos, sendo selecionados somente 16 artigos para leitura na íntegra e provável inclusão na revisão integrativa. 
A seleção dos artigos foi baseada no título e resumo, sendo realizada por três revisores que levaram em consideração os critérios de inclusão e exclusão da revisão. Em casos de divergências na análise de seleção, foi consultado um quarto revisor para assegurar o cumprimento dos requisitos do estudo.

A atividade antileishmania, antimalárica e antitrypanossoma das espécies de Casearia in vitro foi considerada ativa quando a $\mathrm{CI}_{50}$ (Concentração Inibitória Média) foi menor ou igual a $100 \mu \mathrm{g} / \mathrm{mL}$, moderadamente ativo com $\mathrm{CI}_{50}$ entre 101 - 200 $\mu \mathrm{g} / \mathrm{mL}$ e inativo com $\mathrm{CI}_{50}$ maior que $200 \mu \mathrm{g} / \mathrm{mL}$ (Mota et al., 2015; Brígido et al., 2020).

Quanto a citotoxicidade in vitro das espécies de Casearia, foi considerada muito tóxico uma $\mathrm{CC}_{50}$ (Concentração Citotóxica 50\%) $\leq 100 \mu \mathrm{g} / \mathrm{mL}$, e moderadamente tóxico uma $\mathrm{CC}_{50}$ entre 101 a $500 \mu \mathrm{g} / \mathrm{mL}$ e não tóxico uma $\mathrm{CC}_{50}>500 \mu \mathrm{g} / \mathrm{mL}$ (Galucio 2014, Brígido et al., 2020). O índice de seletividade (IS) foi calculado pela razão entre $\mathrm{CC}_{50}$ e $\mathrm{CI}_{50}$ (Nakamura et al., 2006).

\section{Resultados e Discussão}

Doenças parasitárias como: leishmaniose, malária e doença de chagas estão incluídas na lista de doenças negligenciadas pela indústria farmacêutica pelo fato de que a terapia medicamentosa não trará um bom retorno financeiro, tendo em vista que a população atingida é carente, o que ocasiona os índices elevados de casos e mortes anuais dessas doenças (Belaunzarán, 2015). Desta forma, a pesquisa e desenvolvimento de novas alternativas terapêuticas são de grande importância para reverter o cenário atual, dentre elas, o estudo com plantas medicinais vem ganhando cada vez mais destaque, pois as espécies vegetais possuem substâncias farmacologicamente ativas.

Neste contexto, nosso estudo avaliou, por meio de uma revisão de literatura, a atividade antiparasitária de espécies do gênero Casearia. Essas espécies são utilizadas na medicina tradicional como analgésicos, anti-inflamatórios, antiviral, antiulceroso e também é usada em infecções e lesões na pele e mucosas (Howard, 1989). Pode-se acrescentar também que possui outras indicações como a neutralização de venenos e a coagulação de sangramentos (Borges, 1997; Borges et al., 2000; Tropical plant database., 2006). Dessas espécies vários metabólitos secundários já foram isolados como: flavonóides (Shaari; Waterman., 1994; Raslan et al., 2002), taninos (Weniger et al., 1978), cumarinas (Talapatra et al., 1982), compostos fenólicos (Raslan et al., 2002; Mosaddik et al., 2007) e terpenóides (Wang et al., 2009; Shaari; Waterman., 1994; Gunasekera et al., 1977), se destacando diterpenos clerodânicos (Vieira junior et al., 2010).

\subsection{Atividade Antileishmania}

$\mathrm{Na}$ avaliação da atividade antileishmania verificou-se que C. sylvestris é a espécie mais estudada (Bou et al., 2014; Mesquita et al., 2005; Moreira et al., 2019; Antinarelli et al., 2015). Dentre os artigos encontrados, todas as amostras desta planta avaliadas frente a diferentes espécies de Leishmania foram ativas, ou seja, apresentaram $\mathrm{CI}_{50}<100 \mu \mathrm{g} / \mathrm{mL}$ (Tabela 1). No entanto, todas as amostras apresentaram elevada toxicidade $\left(\mathrm{CC}_{50}<100 \mu \mathrm{g} / \mathrm{mL}\right)$, resultando em amostras com baixa seletividade. Dentre as amostras com maior atividade antipromastigota, os extratos de hexano da madeira do caule e da casca do caule, além do extrato etanólico da casca da raiz de C. sylvestris mostram ser os mais promissores, com $\mathrm{CI}_{50}<1 \mu \mathrm{g} / \mathrm{mL}$, apresentando elevada atividade. Porém, a citotoxicidade dessas 3 amostras não foi avaliada. Desta forma não foi possível determinar o índice de seletividade (Tabela 1).

A C. sylvestris pode ser encontrada em todo o mundo e é popularmente conhecida no Brasil como guaçatonga, cafezinho-do-mato ou erva-de-lagarto (Ferreira et al., 2011). Ela é utilizada na medicina popular como anti-inflamatório, antiúlcera, anti-pirético (Ferreira et al., 2011). Seus principais metabólitos secundários são terpenos, esteróis, taninos e flavonoides. Dentre os terpenos destacam-se os diterpenos clerodanos, provavelmente os principais responsáveis pelos efeitos biológicos da espécie. Estes compostos possuem alta hidrofobicidade, o que está relacionado à sua facilidade para atravessar a membrana celular e interagir com o meio intracelular (Ferreira et al., 2010). 


\begin{tabular}{|c|c|c|c|c|c|c|c|c|c|c|}
\hline \multirow[t]{2}{*}{$\begin{array}{l}\text { Espécie } \\
\text { vegetal }\end{array}$} & \multirow[t]{2}{*}{$\begin{array}{l}\text { Parte da } \\
\text { planta }\end{array}$} & \multirow[t]{2}{*}{ Amostra } & \multicolumn{3}{|c|}{ Atividade Antileishmania (CI50 $\mu \mathrm{g} / \mathrm{mL}$ ) } & \multicolumn{2}{|c|}{$\begin{array}{c}\text { Citotoxicidade (CC50 } \\
\mu \mathrm{g} / \mathrm{mL})\end{array}$} & \multirow[t]{2}{*}{$\begin{array}{c}\text { Classificação da } \\
\text { Atividade } \\
\end{array}$} & \multirow[t]{2}{*}{ IS } & \multirow[t]{2}{*}{ Referência } \\
\hline & & & Promastigota & Amastigota & Espécie & $\mathrm{CC}_{50}$ & Células & & & \\
\hline C. javitensis & $\begin{array}{c}\text { Casca do } \\
\text { tronco }\end{array}$ & Etanol (E) & $>200$ & ND & L. amazonensis & $\begin{array}{c}88.77 \pm \\
2.8\end{array}$ & $\begin{array}{l}\text { Macrófagos } \\
\text { THP-1 } \\
\end{array}$ & $\begin{array}{c}\text { Inativo e } \\
\text { Citotóxico } \\
\end{array}$ & ND & Silva et al., 2020 \\
\hline C. javitensis & $\begin{array}{c}\text { Casca do } \\
\text { tronco }\end{array}$ & Hexano $(\mathrm{F})$ & $116.6 \pm 0.9$ & ND & L. amazonensis & $\begin{array}{c}333.4 \pm \\
3.2 \\
\end{array}$ & Macrófagos & $\begin{array}{c}\text { Mod. Ativo e } \\
\text { Mod. Citotóxico }\end{array}$ & 2,85 & Silva et al., 2020 \\
\hline C. javitensis & $\begin{array}{c}\text { Casca do } \\
\text { tronco }\end{array}$ & Diclorometano $(F)$ & 59,38 & ND & L. amazonensis & $\begin{array}{c}241.2 \pm \\
1.9 \\
\end{array}$ & THP-1 & $\begin{array}{l}\text { Ativo e Mod. } \\
\text { Citotóxico }\end{array}$ & 4,06 & Silva et al., 2020 \\
\hline C. javitensis & $\begin{array}{c}\text { Casca do } \\
\text { tronco }\end{array}$ & Acetato de etila (F) & $>200$ & ND & L. amazonensis & $30.5 \pm 5.3$ & Macrófagos & $\begin{array}{c}\text { Inativo e } \\
\text { Citotóxico } \\
\end{array}$ & ND & Silva et al., 2020 \\
\hline C. javitensis & $\begin{array}{c}\text { Casca do } \\
\text { tronco }\end{array}$ & Metanol (F) & $>200$ & ND & L. amazonensis & $\begin{array}{c}101.4 \pm \\
3.1 \\
\end{array}$ & THP-1 & $\begin{array}{c}\text { Inativo e Mod. } \\
\text { citotóxico }\end{array}$ & ND & Silva et al., 2020 \\
\hline C. sylvestris & Folhas & Óleo essencial & $29.8 \pm 1.1$ & $14.0 \pm 5.9$ & L. amazonensis & $40,8 \pm 4,5$ & $\begin{array}{c}\text { Macrófagos } \\
\text { J774-A1 }\end{array}$ & Ativo e Citotóxico & $\begin{array}{l}1,3(\mathrm{P}) \\
2,9(\mathrm{~A})\end{array}$ & Moreira et al., 2019 \\
\hline C. sylvestris & Folhas & Metanol (E) & 5 & 1,3 & L. braziliensis & 5,2 & $\begin{array}{c}\text { Macrófagos } \\
\text { Murinos }\end{array}$ & Ativo e Citotóxico & $\begin{array}{c}1,04(\mathrm{P}) \\
4(\mathrm{~A})\end{array}$ & $\begin{array}{c}\text { Antinarelli et al., } \\
2015\end{array}$ \\
\hline C. sylvestris & Folhas & Metanol (E) & 5,4 & ND & L. amazonensis & 5,2 & $\begin{array}{c}\text { Macrófagos } \\
\text { Murinos }\end{array}$ & Ativo e Citotóxico & 0,96 & $\begin{array}{c}\text { Antinarelli et al., } \\
2015 \\
\end{array}$ \\
\hline C. sylvestris & Folhas & Metanol (E) & 7,7 & ND & L. major & 5,2 & $\begin{array}{c}\text { Macrófagos } \\
\text { Murinos }\end{array}$ & Ativo e Citotóxico & 0,67 & $\begin{array}{c}\text { Antinarelli et al., } \\
2015 \\
\end{array}$ \\
\hline C. sylvestris & Folhas & Metanol (E) & 5,2 & ND & L. chagasi & 5,2 & $\begin{array}{c}\text { Macrófagos } \\
\text { Murinos }\end{array}$ & Ativo e Citotóxico & 1,04 & $\begin{array}{c}\text { Antinarelli et al., } \\
2015 \\
\end{array}$ \\
\hline C. sylvestris & Folhas & Casearina $\mathrm{A}$ & 6,34 & ND & L. chagasi & 2,62 & $\begin{array}{l}\text { Células } \\
\text { Murinos } \\
\end{array}$ & Ativo e citotóxico & 0,41 & Bou et al., 2014 \\
\hline C. sylvestris & Folhas & Casearina B & 9,48 & ND & L. chagasi & 13,76 & $\begin{array}{l}\text { Células } \\
\text { Murinos }\end{array}$ & Ativo e Citotóxico & 1,45 & Bou et al., 2014 \\
\hline C. sylvestris & Folhas & Casearina $\mathbf{J}$ & 4,45 & ND & L. chagasi & 1,46 & $\begin{array}{l}\text { Células } \\
\text { Murinos }\end{array}$ & Ativo e Citotóxico & 0,32 & Bou et al., 2014 \\
\hline C. sylvestris & Folhas & Casearina $\mathrm{G}$ & 5,93 & ND & L. chagasi & 7,54 & $\begin{array}{l}\text { Células } \\
\text { Murinos }\end{array}$ & Ativo e Citotóxico & 1,27 & Bou et al., 2014 \\
\hline C. sylvestris & Folhas & Hexano (E) & $3,7 \pm 0.3$ & ND & L. donovanii & ND & - & Ativo & - & $\begin{array}{l}\text { Mesquita et al., } \\
2005\end{array}$ \\
\hline C. sylvestris & Casca do & Etanol (E) & $11.4 \pm 0.2$ & ND & L. donovanii & ND & - & Ativo & - & Mesquita et al., \\
\hline
\end{tabular}


Research, Society and Development, v. 10, n. 7, e34410716743, 2021

(CC BY 4.0) | ISSN 2525-3409 | DOI: http://dx.doi.org/10.33448/rsd-v10i7.16743

\begin{tabular}{|c|c|c|c|c|c|c|c|c|c|c|}
\hline & caule & & & & & & & & & 2005 \\
\hline C. sylvestris & $\begin{array}{c}\text { Casca do } \\
\text { caule }\end{array}$ & Hexano (E) & $0.2 \pm 0.0$ & ND & L. donovanii & ND & - & Ativo & - & $\begin{array}{l}\text { Mesquita et al., } \\
2005\end{array}$ \\
\hline C. sylvestris & $\begin{array}{l}\text { Madeira do } \\
\text { caule }\end{array}$ & Hexano (E) & $0.3 \pm 0.0$ & ND & L. donovanii & ND & - & Ativo & - & $\begin{array}{c}\text { Mesquita et al., } \\
2005\end{array}$ \\
\hline C. sylvestris & $\begin{array}{c}\text { Casca da } \\
\text { raiz }\end{array}$ & Etanol (E) & $0.1 \pm 0.0$ & ND & L. donovanii & ND & - & Ativo & - & $\begin{array}{c}\text { Mesquita et al., } \\
2005\end{array}$ \\
\hline C. sylvestris & $\begin{array}{c}\text { Casca da } \\
\text { raiz }\end{array}$ & Hexano (E) & $1.0 \pm 0.1$ & ND & L. donovanii & ND & - & Ativo & - & $\begin{array}{c}\text { Mesquita et al., } \\
2005\end{array}$ \\
\hline C. sylvestris & $\begin{array}{c}\text { Madeira da } \\
\text { raiz }\end{array}$ & Etanol (E) & $5.0 \pm 0.1$ & ND & L. donovanii & ND & - & Ativo & - & $\begin{array}{c}\text { Mesquita et al., } \\
2005\end{array}$ \\
\hline C. sylvestris & $\begin{array}{c}\text { Madeira da } \\
\text { raiz }\end{array}$ & Hexano (E) & $11.4 \pm 0.2$ & ND & L. donovanii & ND & - & Ativo & - & $\begin{array}{c}\text { Mesquita et al., } \\
2005\end{array}$ \\
\hline C. sylvestris & Frutos & Etanol (E) & $4.9 \pm 0.2$ & ND & L. donovanii & ND & - & Ativo & - & $\begin{array}{c}\text { Mesquita et al., } \\
2005\end{array}$ \\
\hline C. sylvestris & $\begin{array}{c}] \\
\text { Frutos }\end{array}$ & Hexano (E) & $9.5 \pm 0.6$ & ND & L. donovanii & ND & - & Ativo & - & $\begin{array}{c}\text { Mesquita et al., } \\
2005\end{array}$ \\
\hline C. arborea & Folhas & Tricina & $>100$ & 18,7 & L. chagasi & $>400$ & $\begin{array}{l}\text { Células } \\
\text { Murinos }\end{array}$ & $\begin{array}{l}\text { Ativo e não } \\
\text { citotóxico }\end{array}$ & $\begin{array}{c}>4(\mathrm{P}) \\
21,39(\mathrm{~A})\end{array}$ & Santos et al., 2017 \\
\hline C. arborea & Folhas & $\begin{array}{c}\text { 1',6'-di-O- } \beta \text {-d- } \\
\text { vaniloil } \\
\text { glucopiranosídeo }\end{array}$ & $>100$ & $>100$ & L. chagasi & $>400$ & $\begin{array}{l}\text { Células } \\
\text { Murinos }\end{array}$ & Inativo & ND & Santos et al., 2017 \\
\hline C. arborea & Folhas & ácido vanílico & $>100$ & $>100$ & L. chagasi & $>400$ & $\begin{array}{l}\text { Células } \\
\text { Murinos }\end{array}$ & Inativo & ND & Santos et al., 2017 \\
\hline
\end{tabular}

Legenda: E - Extrato; F - Fração; Mod. - Moderadamente; NCTC - Células murinos; ND - Não determinado; A - amastigota; P - Promastigota; J774-A1 - Macrófagos murinos; THP-1 linhagem celular de leucemia monocítica aguda; IS - Índice de Seletividade.

Fonte: Oliveira MS et al. (2021).

Foi observado uma carência de estudos de atividade contra a forma amastigota de Leishmania, ou seja, a maioria dos estudos foram realizados apenas contra a forma promastigota. Em apenas 2 estudos com C. sylvestris foi avaliada a atividade antiamastigota (Tabela 1). No estudo de Antinarelli et al., (2015), o extrato de metanol das folhas de $C$. sylvestris se mostrou muito ativo $\left(\mathrm{CI}_{50}=1,3 \mu \mathrm{g} / \mathrm{mL}\right)$ e no estudo de Moreira et al., (2019), o óleo essencial de $C$. sylvestris também se mostrou ativo $\left(\mathrm{CI}_{50}=14 \mu \mathrm{g} / \mathrm{mL}\right)$. No entanto, as duas amostras apresentaram elevada citotoxicidade $\left(\mathrm{CC}_{50}\right.$ de 5,2 e 40,8 $\mu \mathrm{g} / \mathrm{mL}$, respectivamente). 
Estudos fitoquímicos demonstram que o óleo essencial obtido de C. sylvestris já foram isolados sesquiterpenos $\alpha$ zingibereno, E-cariofileno, germacreno D e biciclogermacreno (Santos et al., 2008). O mecanismo de ação frente a Leishmania pode estar relacionado com a ruptura da membrana plasmática do parasito provocada pelos sesquiterpenos, que interagem com os componentes lipofílicos da membrana (Di Pascua et al., 2007). Além disso, no citoplasma pode ocorrer a despolarização das membranas mitocondriais por conta destes compostos, levando a apoptose ou necrose celular (Tariku et al., 2010; Armstrong, 2006).

Além da avaliação antileishmania de extratos e frações, verificamos que quatro terpenos (casearinas A, B, J e G) isolados de C. sylvestris e um flavonoide (tricina) isolado das folhas de C. arborea também já foram avaliados frente a promastigotas de L. chagasi e mostraram atividade (Tabela 1). As casearinas A, B, J e G apresentaram elevada atividade $\left(\mathrm{CI}_{50}<10 \mu \mathrm{g} / \mathrm{mL}\right)$, porém não foram seletivas, apresentando elevada toxicidade contra macrófagos $\left(\mathrm{CC}_{50}<15 \mu \mathrm{g} / \mathrm{mL}\right)$. Por outro lado, o flavonoide tricina se mostrou ativo e não citotóxico (Tabela 1).

Vários flavonoides apresentam atividade antileishmania, a exemplo da quercetina e luteolina. A quercetina é capaz de provocar a morte da espécie L. amazonensis, fazendo com que aumente a produção de espécies reativas de oxigênio, desorganizando o potencial mitocondrial (Fonseca, 2014). Outro alvo da quercetina é a enzima arginase que é responsável pela produção das poliaminas (Da Silva et al., 2012). Sua inativação e consequente redução na produção de polimaminas impede a correta clivagem do DNA para duplicação durante a mitose, levando assim à apoptose celular (Mittra et al., 2000).

Outra espécie que apresentou atividade nos artigos pesquisados foi C. javitensis, conhecida popularmente como caneleira ou mata-calado, possui porte arbóreo, folhas oblongas, com a base obtusa e borda do limbo serrilhada (Flora Brasilensis, 1871). Na medicina tradicional a espécie é usada para o tratamento de infecções e picada de cobras (Prieto et al., 2013; Silva et al., 2008). Estudos fitoquímicos mostram que a espécie possuí uma presença marcante de terpenos, ácidos hexanóico, capróico (Xia et al., 2015; Pietro et al., 2013), glicosídeos fenólicos, esteróides e flavonóides. Em sua fração de diclorometano é possível encontrar triterpeno friedelina, $\beta$-friedelanol e esteróide $\beta$-sitosterol (Wyrepkowski, 2010).

Das cinco amostras obtidas da casca do tronco de C. javitensis, apenas a fração de hexano $\left(\mathrm{CI}_{50}=116,6 \mu \mathrm{g} / \mathrm{mL}\right)$ e a fração de diclorometano $\left(\mathrm{CI}_{50}=59,38 \mu \mathrm{g} / \mathrm{mL}\right)$ apresentaram atividade, sendo que a fração de diclorometano apresentou melhor valor de seletividade (IS=4,06; Tabela 1). Segundo Silva et al., (2020), a atividade antileishmania da fração de diclorometano obtida de $C$. javitensis pode estar associada a presença de flavonoides, visto que estes metabólitos foram identificados na espécie.

\subsection{Atividade Antimalárica}

Avaliando a atividade antimalárica de espécies de Casearia verificamos uma escassez de estudos, sendo que apenas três estudos demostram o potencial de espécies do gênero (C. sylvestris, C. elliptica e C. coriaceae) frente a cepas sensíveis de Plasmodium falciparum (Tabela 2). Todos os 11 extratos avaliados obtidos de diferentes partes das 3 plantas se mostraram ativos $\left(\mathrm{CI}_{50}<100 \mu \mathrm{g} / \mathrm{mL}\right)$. Os extratos obtidos com hexano de C. sylvestris, apresentaram maior atividade, ganhando destaque o extrato de hexano da madeira do caule $\left(\mathrm{CI}_{50}=0,9 \mu \mathrm{g} / \mathrm{mL}\right)$, o extrato de hexano da casca do caule $\left(\mathrm{CI}_{50}=1,0 \mu \mathrm{g} / \mathrm{mL}\right)$, o extrato de hexano da casca da raiz $\left(\mathrm{CI}_{50}=1,2 \mu \mathrm{g} / \mathrm{mL}\right)$ e o extrato de hexano das folhas $\left(\mathrm{CI}_{50}=1,3 \mu \mathrm{g} / \mathrm{mL}\right)$. Apenas o extrato de hexano da madeira do caule da C. sylvestris foi avaliado quanto a citotoxicidade, e verificou-se que se trata de uma amostra citotóxica, apresentando baixa seletividade (Tabela 2). 
Tabela 2: Atividade antimalárica de espécies de Casearia.

\begin{tabular}{|c|c|c|c|c|c|c|c|c|c|c|}
\hline \multirow[t]{2}{*}{$\begin{array}{l}\text { Espécie } \\
\text { vegetal }\end{array}$} & \multirow[t]{2}{*}{$\begin{array}{c}\text { Parte da } \\
\text { planta }\end{array}$} & \multirow[t]{2}{*}{ Amostra } & \multicolumn{3}{|c|}{ Atividade antimalárica $\left(C_{50} \mu \mathrm{g} / \mathrm{mL}\right)$} & \multicolumn{2}{|c|}{$\begin{array}{c}\begin{array}{c}\text { Citotoxicidade }\left(\mathrm{CC}_{50}\right. \\
\mu \mathrm{g} / \mathrm{mL})\end{array} \\
\end{array}$} & \multirow[t]{2}{*}{$\begin{array}{l}\text { Classificação } \\
\text { da atividade }\end{array}$} & \multirow[t]{2}{*}{ IS } & \multirow[t]{2}{*}{ Referência } \\
\hline & & & Sensível & Resistente & Espécie & $\mathrm{CC}_{50}$ & Células & & & \\
\hline C. elliptica & Folha & Etanol (E) & 9 & ND & $\begin{array}{l}\text { P. falciparum } \\
\text { 3D7 }\end{array}$ & ND & - & Ativo & ND & $\begin{array}{l}\text { Simonsen et } \\
\text { al., } 2000\end{array}$ \\
\hline C. elliptica & Tronco & Etanol (E) & 33 & ND & $\begin{array}{l}\text { P. falciparum } \\
\text { 3D7 } \\
\end{array}$ & ND & - & Ativo & ND & $\begin{array}{c}\text { Simonsen et } \\
\text { al., } 2000\end{array}$ \\
\hline C. coriaceae & cascas & Etanol (E) & $4.24 \pm 2.2$ & ND & $\begin{array}{c}\text { P. falciparum } \\
\text { 3D7 }\end{array}$ & ND & - & Ativo & ND & $\begin{array}{c}\text { Ledoux et al., } \\
2018\end{array}$ \\
\hline C. coriaceae & Folhas & Etanol (E) & $3.62 \pm 1.02$ & ND & $\begin{array}{l}\text { P. falciparum } \\
\text { 3D7 }\end{array}$ & ND & - & Ativo & ND & $\begin{array}{c}\text { Ledoux et al., } \\
2018\end{array}$ \\
\hline C. sylvestris & Folhas & Hexano (E) & $1,3 \pm 0,1$ & ND & $\begin{array}{l}\text { P. falciparum } \\
\text { 3D7 }\end{array}$ & ND & - & Ativo & ND & $\begin{array}{c}\text { Mesquita et } \\
\text { al., } 2007\end{array}$ \\
\hline C. sylvestris & $\begin{array}{l}\text { Madeira do } \\
\text { caule }\end{array}$ & Hexano (E) & $0,9 \pm 0,2$ & ND & P. falciparum & $1,6 \pm 0,2$ & MRC-5 & $\begin{array}{c}\text { Ativo e } \\
\text { citotóxico }\end{array}$ & 1,73 & $\begin{array}{c}\text { Mesquita et } \\
\text { al., } 2007\end{array}$ \\
\hline C. sylvestris & $\begin{array}{l}\text { Madeira do } \\
\text { caule }\end{array}$ & Hexano (E) & $0,9 \pm 0,2$ & ND & P. falciparum & $1,7 \pm 0,9$ & L-6 & $\begin{array}{l}\text { Ativo e } \\
\text { citotóxico }\end{array}$ & 1,85 & $\begin{array}{c}\text { Mesquita et } \\
\text { al., } 2007\end{array}$ \\
\hline C. sylvestris & $\begin{array}{c}\text { Casca da } \\
\text { raiz }\end{array}$ & Hexano (E) & $1,2 \pm 0,4$ & ND & P. falciparum & ND & - & Ativo & ND & $\begin{array}{c}\text { Mesquita et } \\
\text { al., } 2007\end{array}$ \\
\hline C. sylvestris & $\begin{array}{c}\text { Casca da } \\
\text { raiz }\end{array}$ & Etanol (E) & $7,7 \pm 1,1$ & ND & P. falciparum & ND & - & Ativo & ND & $\begin{array}{c}\text { Mesquita et } \\
\text { al., } 2007\end{array}$ \\
\hline C. sylvestris & $\begin{array}{c}\text { Casca do } \\
\text { caule }\end{array}$ & Hexano (E) & $1,0 \pm 0,4$ & ND & P. falciparum & ND & - & Ativo & ND & $\begin{array}{c}\text { Mesquita et } \\
\text { al., } 2007\end{array}$ \\
\hline C. sylvestris & $\begin{array}{l}\text { Madeira da } \\
\text { raiz }\end{array}$ & Hexano (E) & $2,3 \pm 0,5$ & ND & P. falciparum & ND & - & Ativo & ND & $\begin{array}{c}\text { Mesquita et } \\
\text { al., } 2007\end{array}$ \\
\hline
\end{tabular}

Legenda: E - Extrato; Mod. - Moderadamente; MRC-5 - Células pulmonares embrionárias diplóides humanas; L-6 - Células derivadas de mioblastos; IS - Índice de Seletividade; 3D7 Sensível a cloroquina.

Fonte: Oliveira MS et al. (2021). 
Diversos estudos mostram que C. sylvestris é rica em casearinas e diterpenos, conhecidos por sua atividade citotóxica contra células V-79 (fibroblasto de pulmão permanente) e epidermóide oral humana de carcinoma (Morita et al., 1991; Oberlies et al., 2002). Além destes compostos, já foram identificados nesta espécie compostos fenólicos como flavonoides e derivados do ácido gálico, sequiterpenos componentes do óleo essencial, e já foram isolados mais de 30 diterpenos clerodânicos, sendo 28 típicos do gênero Casearia, como as casearinas A-X e a caseargrewiina F, usualmente isolados das folhas (Santos et al., 2010; Ferreira et al., 2011). Dentre estes compostos, os diterpenos clerodânicos são os que apresentam uma ação antimalárica mais promissora (Kanokmedhakul et al., 2005).

Outros estudos da atividade antimalárica de terpenos demostram que esses metabólitos são promissores para tal atividade. Sequiterpenos bisabolanos p-mentânicos isolados das partes aéreas da Artemisia persica se mostraram ativas quando testadas in vitro frente a uma cepa resistente (K1) de Plasmodium falciparum, mostraram excelente atividade antiplasmódica com $\mathrm{CI}_{50}$ de 2,8 $\mu \mathrm{g} / \mathrm{mL}$ (Moradi-Afrapoli et al., 2013). Vale ressaltar que, uma potente droga antimalárica é o arteether, derivada da artemisinina, um sequiterpeno lactona isolado da Artemisia аппиа (Klayman, 1985, Van Agtmael et al., 1999; Graul, 2001).

\subsection{Atividade Antitrypanossoma}

Em relação à atividade antitrypanossoma de espécies de Casearia, foram encontrados apenas dois estudos (Tabela 3). As casearinas A, B, J e G, isoladas das folhas de C. sylvestris apresentaram grande potencial contra a forma tripomastigota de T. cruzi, com $\mathrm{CI}_{50}<3,0 \mu \mathrm{g} / \mathrm{mL}$. Porém, apresentaram elevada toxicidade contra macrófagos $\left(\mathrm{CC}_{50}<15 \mu \mathrm{g} / \mathrm{mL}\right)$, sendo que a casearina $\mathrm{B}$ foi a que apresentou melhor valor de seletividade (IS $=4,96$ ). 
Tabela 3: Atividade antitrypanossoma de espécies de Casearia.

\begin{tabular}{|c|c|c|c|c|c|c|c|c|c|c|}
\hline \multirow[t]{2}{*}{$\begin{array}{l}\text { Espécie } \\
\text { vegetal }\end{array}$} & \multirow[t]{2}{*}{$\begin{array}{c}\text { Parte da } \\
\text { planta }\end{array}$} & \multirow[t]{2}{*}{ Amostra } & \multicolumn{3}{|c|}{ Atividade antitrypanossoma $\left(\mathrm{CI}_{50} \mu \mathrm{g} / \mathrm{mL}\right)$} & \multicolumn{2}{|c|}{$\begin{array}{c}\text { Citotoxicidade (CC50 } \\
\mu \mathrm{g} / \mathrm{mL})\end{array}$} & \multirow[t]{2}{*}{$\begin{array}{l}\text { Classificação } \\
\text { da atividade }\end{array}$} & \multirow[t]{2}{*}{ IS } & \multirow[t]{2}{*}{ Referência } \\
\hline & & & Amastigota & Tripomastigota & Espécie & $\mathrm{CC}_{50}$ & Células & & & \\
\hline C. sylvestris & Folhas & Casearina $\mathrm{A}$ & ND & 1.37 & T. cruzi & 2.62 & $\begin{array}{l}\text { Células } \\
\text { Murinos }\end{array}$ & Ativo & 1.91 & $\begin{array}{l}\text { Bou et al., } \\
2014\end{array}$ \\
\hline C. sylvestris & Folhas & Casearina B & ND & 2.77 & T. cruzi & 13.76 & $\begin{array}{l}\text { Células } \\
\text { Murinos }\end{array}$ & Ativo & 4.96 & $\begin{array}{l}\text { Bou et al., } \\
2014\end{array}$ \\
\hline C. sylvestris & Folhas & Casearina $\mathrm{J}$ & ND & 0.53 & T. cruzi & 1.46 & $\begin{array}{l}\text { Células } \\
\text { Murinos }\end{array}$ & Ativo & 2.75 & $\begin{array}{l}\text { Bou et al., } \\
2014\end{array}$ \\
\hline C. sylvestris & Folhas & Casearina $\mathrm{G}$ & ND & 1.57 & T. cruzi & 7.54 & $\begin{array}{l}\text { Células } \\
\text { Murinos }\end{array}$ & Ativo & 4.80 & $\begin{array}{l}\text { Bou et al., } \\
2014\end{array}$ \\
\hline C. sylvestris & Folhas & Hexano (E) & $3.40 \pm 0.35$ & ND & T. cruzi & ND & - & Ativo & ND & $\begin{array}{l}\text { Mesquita et } \\
\text { al., } 2005\end{array}$ \\
\hline C. sylvestris & $\begin{array}{c}\text { Casca do } \\
\text { caule }\end{array}$ & Hexano (E) & $0.44 \pm 0.02$ & ND & T. cruzi & ND & - & Ativo & ND & $\begin{array}{l}\text { Mesquita et } \\
\text { al., } 2005\end{array}$ \\
\hline C. sylvestris & $\begin{array}{l}\text { Madeira } \\
\text { do caule }\end{array}$ & Hexano(E) & $0.44 \pm 0.05$ & ND & T. cruzi & ND & & Ativo & ND & $\begin{array}{l}\text { Mesquita et } \\
\text { al., } 2005\end{array}$ \\
\hline C. sylvestris & $\begin{array}{c}\text { Casca da } \\
\text { raiz } \\
\end{array}$ & Etanol (E) & $5.6 \pm 0.4$ & ND & T. cruzi & ND & - & Ativo & ND & $\begin{array}{l}\text { Mesquita et } \\
\text { al., } 2005\end{array}$ \\
\hline C. sylvestris & $\begin{array}{c}\text { Casca da } \\
\text { raiz }\end{array}$ & Hexano (E) & $0.3 \pm 0.04$ & ND & T. cruzi & ND & - & Ativo & ND & $\begin{array}{l}\text { Mesquita et } \\
\text { al., } 2005\end{array}$ \\
\hline C. sylvestris & $\begin{array}{l}\text { Madeira } \\
\text { da raiz }\end{array}$ & Hexano (E) & $0.86 \pm 0.05$ & ND & T. cruzi & ND & - & Ativo & ND & $\begin{array}{l}\text { Mesquita et } \\
\text { al., } 2005\end{array}$ \\
\hline
\end{tabular}

Legenda: ND - não determinado; NCTC - Células murinos; IS - Índice de Seletividade.

Fonte: Oliveira MS et al. (2021) 
Outras seis amostras obtidas de diferentes partes de C. sylvestris também foram avaliadas contra T. cruzi, mas agora contra a forma intracelular (amastigota). Observou-se que quatro amostras obtidas com hexano apresentaram potencial de inibir o crescimento de amastigotas, com $\mathrm{CI}_{50}<1,0 \mu \mathrm{g} / \mathrm{mL}$ (Tabela 3). Apesar da elevada atividade, estudos de toxicidade dessas amostras não foram realizados até o momento, portanto, não se sabe se são seletivas.

$\mathrm{Na}$ literatura já foi descrito diversos resultados da atividade de terpenos frente a formas tripomastigotas de T. cruzi, entre eles o ácido caurenóico isolado de Mikania obtusata, Asteraceae (Alves et al., 1995), Xylopia frutescens, Anmonaceae (Takahashi et al., 1994) e de Viguiera aspillioides, Asteraceae (Costa et al., 1995), apresentaram 100\% de atividade contra o T. cruzi, na concentração de $1000 \mu \mathrm{g} / \mathrm{mL}$ de sangue contaminado (Takahashi et al., 1994).

O taxol um diterpeno isolado de Taxus brevifolia (Taxaceae) foi avaliado in vitro sobre formas epimastigotas e se mostrou ativo, interferindo na proliferação dos epimastigotas, impedindo que a divisão celular se completasse e permitindo a multiplicação de organelas, outro composto ativo foi o triterpeno tingenoma, também extraído da mesma espécie, se apresentou ativo causando inibição da síntese de proteínas e ácidos nucleicos no T. cruzi (Castro, 1993).

Segundo Marr et al., (2012) a atividade antitrypanossoma de terpernos pode estar relacionada a ruptura parcial da membrana do parasito, levando ao esgotamento dos gradientes iônicos, efluxo de nutrientes e de outros componentes citoplasmáticos, degradação do sistema bioenergético do parasita e então a lise osmótica, causando morte celular.

\section{Conclusão}

Dentre as espécies de Casearia, a C. sylvestris foi a espécie mais estudada quanto a atividade antiparasitária. Na atividade antileishmania, os extratos, frações e substâncias isoladas como os terpenos, obtidas de diferentes espécies de Casearia, apresentaram elevada atividade antipromastigota contra diferentes espécies de Leishmania sp., no entanto, apresentaram elevada citotoxicidade. O flavonóide tricina no entanto, é promissor, tendo em vista o elevado índice de seletividade. Observou-se carência de estudos de atividade contra a forma amastigota de Leishmania. Na atividade antitrypanossoma e antimalárica, os extratos, frações e substâncias isoladas apresentaram de forma geral alta atividade, porém, elevada toxicidade. Sendo assim, novos estudos devem ser realizados, com o intuito de isolar os constituintes fitoquímicos responsáveis por tal atividade e visando melhorar a seletividade destes produtos. Uma alternativa possível são técnicas de formulação utilizando encapsulamento com gorduras vegetais ou animais que, à exemplo da anfotericina lipossomal, podem melhor o índice de seletividade de drogas citotóxicas antiparasitárias (Filippin; Souza, 2006).

\section{Conflito de interesses}

Os autores declaram não haver conflito de interesses.

\section{Agradecimentos}

Os autores expressam seus agradecimentos a Faculdade Cosmopolita.

\section{Referências}

Alvar, J., Vélez, I. D., Bern, C., Herrero, M., Desjeux, P., Cano, J., \& WHO Leishmaniasis Control Team. (2012). Leishmaniasis worldwide and global estimates of its incidence. PloS one, 7(5), e35671. https://doi.org/10.1371/journal.pone.0035671.

Antinarelli, Luciana M. R., Pinto, Nícolas C., Scio, Elita, \& Coimbra, Elaine S. (2015). Antileishmanial activity of some Brazilian plants, with particular reference to Casearia sylvestris. Anais da Academia Brasileira de Ciências, 87(2), 733-742. https://doi.org/10.1590/0001-3765201520140288. 
Armstrong, J. S. (2006). Mitochondrial membrane permeabilization: the sine qua non for cell death. Bioessays, 28(3), 253-260. https://doi.org/10.1002/bies.20370.

Belaunzarán, M. L. (2015). Enfermedad de Chagas: globalización y nuevas esperanzas para su cura. Revista argentina de microbiología,47(2), 85-87. https://doi.org/10.1016/j.ram.2015.04.001.

Borges, M. H. (1997). Ação antipeçonhenta do extrato vegetal de Casearia sylvestris (Inibição da atividade fosfolipase A2, hemorrágica e miotóxica de venenos animais pelo extrato de Casearia sylvestris (FLACOURTIACEAE). Biotecnologia Ciência \& Desenvolvimento, 1(4).

Borges, M. H. (1998). Inibição dos principais efeitos tóxicos causados por venenos animais pelo extrato vegetal de Casearia sylvestris (Flacourtiaceae). http://doi.org/10.14393/ufu.di.1998.26.

Borges, M. H., Jamal, C. M., dos Santos, D. C. M., Raslan, D. S., De Lima, M. E., Bioquímica, T. A. D., \& Imunologia, I. U. (2000). Partial purification of Casearia sylvestris SA. Extract and its anti-PLA2 action. Comp. Biochem. Physiol. Ser. B. B, 127, 21-30.

Bou, D. D, Tempone, A. G, Pinto, É. G., Lago, J. H, \& Sartorelli, P. (2014). Atividade antiparasitária e efeito de casearinas isoladas de Casearia sylvestris na membrana plasmática de Leishmania e Trypanosoma cruzi. Phytomedicine: jornal internacional de fitoterapia e fitofarmacologia, 21 (5), 676-681. https://doi.org/ 10.1016/j.phymed.2014.01.004.

Brasil. Programa Nacional de Prevenção e Controle da Malária. 2007.

Brígido, H. P. C.; Silva e Silva, J. V., Bastos, M. L. C.; Correa-Barbosa, J.; Sarmento, R. M.; Costa, E. V. S.; Marinho, A. M. do R.; Coelho-Ferreira, M. R.; Silveira, F. T.; \& Dolabela, M. F. (2020). Atividade antileishmania de Annona glabra L. (Annonaceae). Revista Eletrônica Acervo Saúde, (57), e3701. https://doi.org/ 10.25248/reas.e3701.2020.

Costa, F. B.; Vichnewisk, W.; Albuquerque, S. Terpenóides bioativos de Viguiera aspillioides Gardn. (Asteraceae) com atividade tripanossomicida. 18 ${ }^{\mathrm{a}}$ Reunião da Sociedade Brasileira de Química, 1995.

da Silva, E. R., Maquiaveli, C., \& Magalhães, P. P. (2012). The leishmanicidal flavonols quercetin and quercitrin target Leishmania (Leishmania) amazonensis arginase. Experimental parasitology, 130(3), 183-188. https://doi.org/10.1016/j.exppara.2012.01.015.

De Castro, S. L. (1993). The challenge of Chagas' disease chemotherapy: an update of drugs assayed against Trypanosoma cruzi. Acta tropica, 53(2), 83-98. https://doi.org/10.1016/0001-706X(93)90021-3.

De Mesquita, M. L., Grellier, P., Mambu, L., De Paula, J. E., \& Espindola, L. S. (2007). In vitro antiplasmodial activity of Brazilian Cerrado plants used as traditional remedies. Journal of Ethnopharmacology, 110(1), 165-170. https://doi.org/ 10.1016/j.jep.2006.09.015. Epub 2006 Sep 23.

Di Pasqua, R., Betts, G., Hoskins, N., Edwards, M., Ercolini, D., \& Mauriello, G. (2007). Membrane toxicity of antimicrobial compounds from essential oils. Journal of agricultural and food chemistry, 55(12), 4863-4870. https://doi.org/10.1021/jf0636465.

Drugs for Neglected Diseases Initiative. (2018). Dndi annual report 2018. dndi.org/wp-content/uplods/2019/07/DNDi_2018_AnnualReport.pdf.

Ferreira, P. M. P., Costa-Lotufo, L. V., Moraes, M. O., Barros, F. W., Martins, A., Cavalheiro, A. J., \& Pessoa, C. (2011). Folk uses and pharmacological properties of Casearia sylvestris: a medicinal review. Anais da Academia Brasileira de Ciências, 83(4), 1373-1384. https://doi.org/10.1590/S000137652011005000040

Ferreira, P.M. P., Santos, A. G., Tininis, A. G., Costa, P.M., Cavalheiro, A. J., Bolzani, V. S., \& Pessoa, C. (2010). A casearina X exibe efeitos citotóxicos em células de leucemia desencadeadas pela apoptose. Interações chemico-biológicas, 188(3), 497-504. https://doi.org/10.1016/j.cbi.2010.08.008.

Filippin, F. B., Souza, L. C. (2006). Eficiência terapêutica das formulações lipídicas de anfotericina B. Revista Brasileira de Ciências Farmacêuticas, 42(2). https://doi.org/10.1590/S1516-93322006000200003.

Flora Brasiliensis. 13, 483-484, 1871. <http://florabrasiliensis.cria.org.br/fviewer>.

Fonseca-Silva, F., Inacio, J. D., Canto-Cavalheiro, M. M., \& Almeida-Amaral, E. E. (2011). Reactive oxygen species production and mitochondrial dysfunction contribute to quercetin induced death in Leishmania amazonensis. PloS one, 6(2), e14666. https://doi.org/10.1371/journal.pone.0014666.

França, T. C., Santos, M. G. D., \& Figueroa-Villar, J. D. (2008). Malária: aspectos históricos e quimioterapia. Química Nova, 31(5), 1271-1278. https://doi.org/10.1590/S0100-40422008000500060.

Gonzaga dos Santos, A., Pinheiro Ferreira, P. M., Magela Vieira Júnior, G., Perez, C. C., Gomes Tininis, A., Silva, G. H., \& Cavalheiro, A. J. (2010). Casearin $\mathrm{X}$, its degradation product and other clerodane diterpenes from leaves of Casearia sylvestris: evaluation of cytotoxicity against normal and tumor human cells. Chemistry \& biodiversity, 7(1), 205-215. https://doi.org/10.1002/cbdv.200800342.

Graul, A. I. (2001). The year's new drugs. Drug news \& perspectives, 14(1), 12-31.

Gunasekera, S. P., Sultanbawa, M. U. S., \& Balasubramaniam, S. (1977). Triterpenes of some species of Flacourtiaceae. Phytochemistry. Disponível em: Triterpenes of some species of Flacourtiaceae (fao.org).

Howard, R. A. (1989). Flora of the Lesser Antilles, Leeward and Windward Islands: Vol. 5: Dicotyledoneae-Part 2 by Richard A. Howard \& Allan J. Bornstein. Arnold Arboretum, Harvard Univ.

Kanokmedhakul, S., Kanokmedhakul, K., Kanarsa, T., \& Buayairaksa, M. (2005). New Bioactive Clerodane Diterpenoids from the Bark of Casearia g rewiifolia. Journal of natural products, 68(2), 183-188. https://doi.org/10.1021/np049757k

Klayman, D. L. (1985). Qinghaosu (artemisinin): an antimalarial drug from China. Science, 228(4703), 1049-1055. https://doi.org/10.1126/science.3887571. 
Ledoux, A., Cao, M., Jansen, O., Mamede, L., Campos, P. E., Payet, B., \& Smadja, J. (2018). Antiplasmodial, anti-chikungunya virus and antioxidant activities of 64 endemic plants from the Mascarene Islands. International journal of antimicrobial agents, 52(5), 622-628. https://doi.org/10.1016/j.ijantimicag.2018.07.017

Marquete, R., \& Vaz, A. M. S. D. F. (2007). The genus Casearia in the state of Rio de Janeiro, Brazil. Rodriguésia,58(4), 705-738. https://doi.org/10.1590/2175-7860200758401

Marr, A. K., McGwire, B. S., \& McMaster, W. R. (2012). Modes of action of Leishmanicidal antimicrobial peptides. Future microbiology, 7(9), 10471059. https://doi.org/10.2217/fmb.12.85.

Mesquita, M. L., Desrivot, J., Bories, C., Fournet, A., Paula, J. E., Grellier, P., \& Espindola, L. S. (2005). Antileishmanial and trypanocidal activity of Brazilian Cerrado plants. Memorias do Instituto Oswaldo Cruz, 100(7), 783-787. https://doi.org/10.1590/s0074-02762005000700019

Mittra, B., Saha, A., Roy Chowdhury, A. et al. Luteolin, an Abundant Dietary Component is a Potent Anti-leishmanial Agent that Acts by Inducing Topoisomerase II-mediated Kinetoplast DNA Cleavage Leading to Apoptosis. Mol Med 6, 527-541 (2000). https://doi.org/10.1007/BF03401792.

Moradi-Afrapoli, F., Ebrahimi, S. N., Smiesko, M., Raith, M., Zimmermann, S., Nadjafi, F., \& Hamburger, M. (2013). Bisabololoxide derivatives from Artemisia persica, and determination of their absolute configurations by ECD. Phytochemistry, 85, 143-152. https://doi.org/10.1016/j.phytochem.2012.08.017.

Moreira, Raquel Regina Duarte, Santos, André Gonzaga dos, Carvalho, Flavio Alexandre, Perego, Caio Humberto, Crevelin, Eduardo José, Crotti, Antônio Eduardo Miller, Cogo, Juliana, Cardoso, Mara Lane Carvalho, \& Nakamura, Celso Vataru. (2019). Antileishmanial activity of Melampodium divaricatum and Casearia sylvestris essential oils on Leishmania amazonensis. Revista do Instituto de Medicina Tropical de São Paulo, 61, e33. Epub July 01, 2019. https://doi.org/10.1590/s1678-9946201961033.

Morita, H., Nakayama, M., Kojima, H., Takeya, K., Itokawa, H., Schenkel, e. P., \& Motidome, M. (1991). Structures and cytotoxic activity relationship of casearins, new clerodane diterpenes from Casearia sylvestris Sw. Chemical and Pharmaceutical Bulletin, 39(3), 693-697. https://doi.org/10.1248/cpb.39.693.

Mosaddik, M. A., Banbury, L., Forster, P., Booth, R., Markham, J., Leach, D., \& Waterman, P. G. (2004). Screening of some Australian Flacourtiaceae species for in vitro antioxidant, cytotoxic and antimicrobial activity. Phytomedicine, 11(5), 461-466. https://doi.org/10.1016/j.phymed.2003.12.001.

Mosaddik, M. A., Forster, P. I., Booth, R., \& Waterman, P. G. (2007). Clerodane diterpenes from the stems of Casearia grewiifolia var. gelonioides (Flacourtiaceae/Salicaceae sensu lato). Biochemical systematics and ecology, 9(35), 631-633. https://doi.org/10.1016/j.bse.2007.03.003.

Oberlies, N. H., Burgess, J. P., Navarro, H. A., Pinos, R. E., Fairchild, C. R., Peterson, R. W., ... \& Wall, M. E. (2002). Novel bioactive clerodane diterpenoids from the leaves and twigs of Casearia sylvestris. Journal of Natural Products, 65(2), 95-99. https://doi.org/10.1021/np010459m.

Prieto, A. M., dos Santos, A. G., Oliveira, A. P. S., Cavalheiro, A. J., Silva, D. H., Bolzani, V. S., ... \& Soares, C. P. (2013). Assessment of the chemopreventive effect of casearin B, a clerodane diterpene extracted from Casearia sylvestris (Salicaceae). Food and chemical toxicology, 53, 153-159. https://doi.org/10.1016/j.fct.2012.11.029.

Raslan, D. S., Jamal, C. M., Duarte, D. S., Borges, M. H., \& De Lima, M. E. (2002). Anti-PLA2 action test of Casearia sylvestris Sw. Bollettino Chimico Farmaceutico, 141(6), 457-460. <https://europepmc.org/article/med/12577517>.

Rassi Jr, A., Rassi, A., \& Marin-Neto, J. A (2010). Doença de Chagas. The Lancet , 375 (9723), 1388-1402. https://doi.org/10.1016/S0140-6736(10)60061-X.

Santos, A. L., Yamamoto, E. S., Passero, L. F. D., Laurenti, M. D., Martins, L. F., Lima, M. L., ... \& Sartorelli, P. (2017). Antileishmanial activity and immunomodulatory effects of tricin isolated from leaves of Casearia arborea (Salicaceae). Chemistry \& biodiversity, 14(5), e1600458. https://doi.org/10.1002/cbdv.201600458.

Santos, A. O., Ueda-Nakamura, T., Dias Filho, B. P., Junior, V. F. V., Pinto, A. C., \& Nakamura, C. V. (2008). Effect of Brazilian copaiba oils on Leishmania amazonensis. Journal of ethnopharmacology, 120(2), 204-208. https://doi.org/10.1590/S0074-02762013000100010.

Santos, L. S., \& Nogemz,T.(1995). A diterpene from Mikania obtusata active on Trypanosoma cruzi. Planta Med, 61, 85-37. https://doi.org/10.1055/s-2006958011.

Shaari, K., \& Waterman, P. G. (1994). Podophyllotoxin-type lignans as major constituents of the stems and leaves of Casearia clarkei. Journal of Natural Products, 57(6), 720-724. https://doi.org/10.1021/np50108a006

Silva, S. L. D., Chaar, J. D. S., Figueiredo, P. D. M. S., \& Yano, T. (2008). Cytotoxic evaluation of essential oil from Casearia sylvestris Sw on human cancer cells and erythrocytes. Acta Amazonica, 38(1), 107-112. https://doi.org/10.1590/S0044-59672008000100012.

Simonsen, H. T., Nordskjold, J. B., Smitt, U. W., Nyman, U., Palpu, P., Joshi, P., \& Varughese, G. (2001). In vitro screening of Indian medicinal plants for antiplasmodial activity. Journal of Ethnopharmacology, 74(2), 195-204. https://doi.org/10.1016/s0378-8741(00)00369-x.

Soares Sobrinho, J. L., Medeiros, F. P. de M., de La Roca, M. F., Silva, K. E. R., Lima, L. N. A., \& Rolim Neto, P. J. (2007). Delineamento de alternativas terapêuticas para o tratamento da doença de Chagas. Revista De Patologia Tropical / Journal of Tropical Pathology, 36(2), 103-118. https://doi.org/10.5216/rpt.v36i2.1783.

Takahashi, J. A., Boaventura, M. A. D., Oliveira, A. B., Chiari, E., \& Vieira, H. S. (1994). Isolamento e atividade tripanossomicida de diterpenos caurânicos de Xylopia frutescens.

Talapatra, S. K., Ganguly, N. C., Goswami, S., \& Talapatra, B. (1983). Chemical constituents of Casearia graveolens: some novel reactions and the preferred molecular conformation of the major coumarin, micromelin. Journal of natural products, 46(3), 401-408. https://doi.org/10.1021/np50027a018

Tariku, Y., Hymete, A., Hailu, A., \& Rohloff, J. (2010). Essential-oil composition, antileishmanial, and toxicity study of Artemisia abyssinica and Satureja punctata ssp. punctata from Ethiopia. Chemistry \& biodiversity, 7(4), 1009-1018. https://doi.org/10.1002/cbdv.200900375. 
Research, Society and Development, v. 10, n. 7, e34410716743, 2021

(CC BY 4.0) | ISSN 2525-3409 | DOI: http://dx.doi.org/10.33448/rsd-v10i7.16743

Tropical Plant Database. (2006)

Van Agtmael, M. A., Eggelte, T. A., \& van Boxtel, C. J. (1999). Artemisinin drugs in the treatment of malaria: from medicinal herb to registered medication. Trends in Pharmacological sciences, 20(5), 199-205. https://doi.org/10.1016/S0165-6147(99)01302-4.

Vieira Júnior, G. M. (2010). Contribuição ao estudo dos metabólitos secundários do gênero Casearia e de algumas de suas atividades biológicas. $<$ http://hdl.handle.net/11449/105774>

Waldman, E. A., \& Sato, A. P. S. (2016). Trajetória das doenças infecciosas no Brasil nos últimos 50 anos: um contínuo desafio. Revista de Saúde Pública , 50 , 68. https://doi.org/10.1590/S1518-8787.2016050000232.

Wang, W., Li, X. C., Ali, Z., \& Khan, I. A. (2009). Two new C13 nor-isoprenoids from the leaves of Casearia sylvestris. Chemical and Pharmaceutical Bulletin, 57(6), 636-638. https://doi.org/10.1248/cpb.57.636

Weniger, B., Haag-Berrurier, M., Rohmer, M., \& Anton, R. (1978). Some constituents of Casearia ilicifolia Vent. Planta Medica, 33(02), 170-172. https://doi.org/10.1055/s-0028-1097371

World Health Organization. (2015) Chagas disease (American trypanosomiasis) http://www.who.int/mediacentre/factsheets/fs340/en/.

World Health Organization. (2017) WHO. Wold malaria report 2017. https://www.who.int/malaria/publications/world-malaria-report-2017.

World Health Organization. (2019). Leishmanione. https://www.who.int/health-topics/leishmaniasis\#tab=tab_1.

World Health Organization. (2019). World malaria report 2019 https://www.who.int/publications/i/item/world-malaria-report-2019.

Wyrepkowski, C. D. C. (2010). Estudo fitoquímico e bioatividade de extratos de casearia javitensis kunth. <https://tede.ufam.edu.br/handle/tede/225>.

Xia, L., Guo, Q., Tu, P., \& Chai, X. (2015). The genus Casearia: a phytochemical and pharmacological overview. Phytochemistry Reviews, 14(1), 99-135. https://doi.org/10.1007/s11101-014-9336-6. 